\title{
Susceptibilidade de Rhodnius neglectus, Rhodnius robustus e Triatoma infestans (Hemiptera, Reduviidae, Triatominae) à infecção por duas cepas de Trypanosoma cruzi (Kinetoplastidae, Trypanosomatidae) utilizando xenodiagnóstico artificial
}

\author{
Susceptibility of Rhodnius neglectus, Rhodnius robustus and Triatoma infestans
} (Hemiptera, Reduviidae, Triatominae) to two Trypanosoma cruzi strains infection (Kinetoplastidae, Trypanosomatidae) using artificial xenodiagnosis

\section{Luciamáre Perinetti Alves Martins', João Aristeu da Rosa², Roberto Esteves Pires Castanho', Guilherme Lopes Sauniti' e Hermano Medeiros Júnior ${ }^{1}$}

Resumo A susceptibilidade de ninfas de $3^{\circ}$ estádio de Rhodnius neglectus, R. robustus e Triatoma infestans às cepas $Y$ e AMJM de Trypanosoma cruzi foi verificada utilizando xenodiagnóstico artificial. Para a leitura do xenodiagnóstico, as fezes dos triatomíneos foram examinadas a cada dois dias, a partir do $5^{\circ}$ até o $31^{\circ}$ dia pós infecção, pela técnica de compressão abdominal. Os resultados mostraram diferenças na susceptibilidade dos triatomíneos para as duas cepas estudadas e o período ótimo de leitura variou do $11^{\circ}$ ao $19^{\circ}$ dias para a cepa $Y$ e do $11^{\circ}$ ao $15^{\circ}$ dias para a cepa AMJM. Também, pôde-se concluir que para a cepa Y, as três espécies de triatomíneos demonstraram boa susceptibilidade, enquanto para a cepa AMJM, a melhor susceptibilidade foi observada com R. neglectus, seguida pelo T. infestans e R. robustus.

Palavras-chaves: Rhodnius neglectus. Rhodnius robustus. Triatoma infestans. Trypanosoma cruzi. Xenodiagnóstico artificial.

Abstract The susceptibility of $3^{\text {rd }}$ instar nymph of Triatominae Rhodnius neglectus, R. robustus and Triatoma infestans to Trypanosoma cruzi $Y$ and AMJM strains was verified using artificial xenodiagnosis. After the accomplishment of the xenodiagnosis, the faeces of the Triatominae were analyzed on two-day intervals from day 5 until day 31 post infection, using the abdominal compression technique. The results showed differences in the susceptibility of the Triatominae for the two strains studied, and the optimal period reading differed from day 11 to day 19 for the $Y$ strain and from day 11 to day 15 for the AMJM strain. For the $Y$ strain, all three Triatominae species showed good susceptibility, whereas in the AMJM strain, the highest susceptibility was observed with $\mathrm{R}$. neglectus, followed by $\mathrm{T}$. infestans and $\mathrm{R}$. robustus.

Key-words: Rhodnius neglectus. Rhodnius robustus. Triatoma infestans. Trypanosoma cruzi. Artificial xenodiagnosis.

A doença de Chagas passa por períodos ou fases, e o diagnóstico laboratorial, tanto parasitológico como imunológico são importantes para detectar a infecção pelo
T. cruzi. Na fase aguda a demonstração parasitológica do $T$. cruzi é facilitada devido à elevada parasitemia, podendo também ser realizada a pesquisa de anticorpos ${ }^{2}$ e 0

\footnotetext{
1.Disciplina de Parasitologia da Faculdade de Medicina de Marília, SP, Brasil;2.Departamento de Ciências Biológicas da Faculdade de Ciências Farmacêuticas, UNESP, Araraquara, SP, Brasil.

Endereço para correspondência: Dra. Luciamáre Perinetti Alves Martins. Disc. de Parasitologia/Faculdade de Medicina de Marília. Av. Monte Carmelo 800, 17519-030, Marília, SP.

Fax: 5514 433-1366/423-4781.

e-mail: luciapam @ famema.br

Recebido para publicação em 19/5/2000.
} 
xenodiagnóstico natural ou artificial ${ }^{426}$. Estando o paciente na fase crônica, o diagnóstico imunológico é o mais usado ${ }^{9}$, podendo-se recorrer à hemocultura ${ }^{6}$, ao xenodiagnóstico natural ou artificial e também à inoculação em animais de laboratório.

O xenodiagnóstico natural foi introduzido por Brumpt $^{3}$ em 1914, e somente em 1940 começou a ser empregado como método de diagnóstico parasitológico da doença de Chagas ${ }^{27}$. Em 1947, Romaña e $\mathrm{Gil}^{16}$, propuseram o xenodiagnóstico artificial, devido algumas desvantagens apresentadas pela técnica natural, por exemplo, a recusa dos pacientes por se deixarem picar pelos barbeiros e o aparecimento de reações alérgicas à saliva dos triatomíneos apresentadas por alguns indivíduos; como podemos citar as reações alérgicas cutâneas causadas pelo Triatoma infestans, espécie de triatomíneo muito utilizada em xenodiagnóstico ${ }^{711}$. Outro triatomíneo responsável por severas reações alérgicas nos indivíduos é o T. rubrofasciata ${ }^{25}$, espécie não utilizada em xenodiagnósticos, mas de importância epidemiológica.

Vários autores ${ }^{121824}$ compararam a eficácia do xenodiagnóstico natural e artificial e concluíram que o xenodiagnóstico artificial pode substituir 0 natural em efetividade.

Assim, com o intuito de melhorar a sensibilidade do xenodiagnóstico, vários pesquisadores, entre eles Perlowagora-Szumlewicz et $\mathrm{al}^{14}$, tem se empenhado na busca de um inseto ideal para ser utilizado nesse exame, pois foi verificado que espécies de triatomíneos de localidades diversas, também demonstravam boa susceptibilidade à infecção por cepas brasileiras de T. cruzi'.

Com o propósito de contribuir para a busca de um inseto ideal a ser utilizado no xenodiagnóstico artificial, assim como avaliar qual o intervalo propício para execução da leitura do xenodiagnóstico artificial, foram utilizados ninfas de $3^{\circ}$ estádio de Rhodnius neglectus, $R$. robustus e Triatoma infestans e as cepas Y e AMJM de T. cruzi.

\section{MATERIAL E MÉTODOS}

Neste estudo utilizou-se as cepas $Y^{23}$ e AMJM $^{17}$ de T.cruzique são mantidas por repiques sucessivos em camundongos Swiss no laboratório de Parasitologia da Faculdade de Ciências Farmacêuticas - UNESP Araraquara.

O sangue utilizado para a realização dos xenodiagnósticos foi colhido em seringa heparinizada $(40 \mu \mathrm{l}$ de heparina sódica para cada $\mathrm{ml}$ de sangue na concentração de $5.000 \mathrm{Ul} / \mathrm{ml}$ ) obtido de camundongos Swiss de 20 dias de idade previamente infectados via intraperitoneal com 5,0 × $10^{4}$ tripomastigotas sanguíneos com a cepa AMJM e com 1,0 x $10^{5}$ tripomastigotas sanguíneos com a cepa $\mathrm{Y}$.

Para cada xenodiagnóstico artificial, utilizouse 120 ninfas de $3^{\circ}$ estádio de cada uma das seguintes espécies de triatomíneo: $R$. neglectus, $R$. robustus e $T$. infestans, sendo que para cada espécie de triatomíneo e cepa de T.cruzi examinada, foram realizados dois xenodiagnósticos, num total de 12 exames ao final do estudo. Seguiuse a técnica proposta por Silva ${ }^{19}$ para a realização do xenodiagnóstico artificial.

As leituras dos xenodiagnósticos foram feitas a partir de triatomíneos alimentados uma única vez, sendo a primeira leitura realizada no $5^{\circ}$ dia após a infecção e as seguintes a cada 48 horas até completar trinta e um dias pós infecção. Como muitos triatomíneos não puderam ser examinados por motivos variados, e sendo possível, nos demais, realizar mais de uma leitura, ao final do estudo foram feitas 2.002 leituras, sendo 1.009 realizadas com a cepa $Y$ e 993 com a cepa AMJM. As dejeções dos triatomíneos foram obtidas através da técnica da compressão abdominal por meio de pinças ${ }^{5}$. Para análise dos resultados foi considerado a percentagem de triatomíneos positivos em cada dia de leitura.

\section{RESULTADOS}

Das 1.009 leituras realizadas em ninfas de 3 estádio alimentadas com o sangue de camundongos infectados com a cepa $Y$, foi encontrado 860 positivos $(85,2 \%)$ e das 993 leituras com a cepa AMJM, $714(71,9 \%)$ foram positivas.

Para a cepa $Y$ de T. cruzi, o melhor período de leitura foi encontrado entre o $11^{\circ}$ e $19^{\circ}$ dias e para a cepa AMJM de T. cruzi entre o $11^{\circ} \mathrm{e}$ $15^{\circ}$ dias.

Os resultados obtidos com as leituras dos xenodiagnósticos artificiais utilizando as cepas Y e AMJM de T. cruzie as espécies $R$. neglectus, $R$. robustus e $T$. infestans estão expressos nas Figuras 1 e 2, respectivamente. 


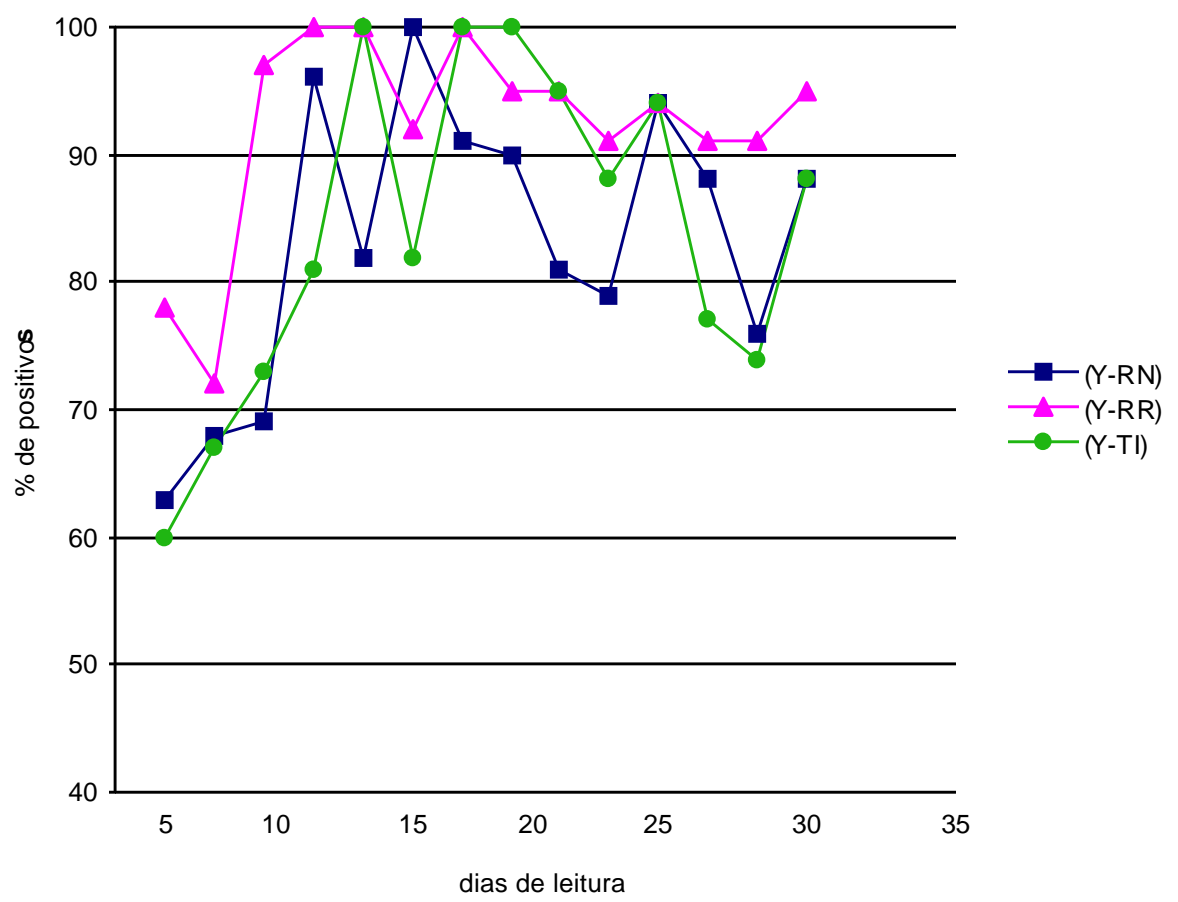

Figura 1 - Curvas médias relativas aos xenodiagnósticos realizados com a cepa Y de Trypanosoma cruzi e os triatomíneos Rhodnius neglectus $(R N)$, Rhodnius robustus (RR) e Triatoma infestans (TI).

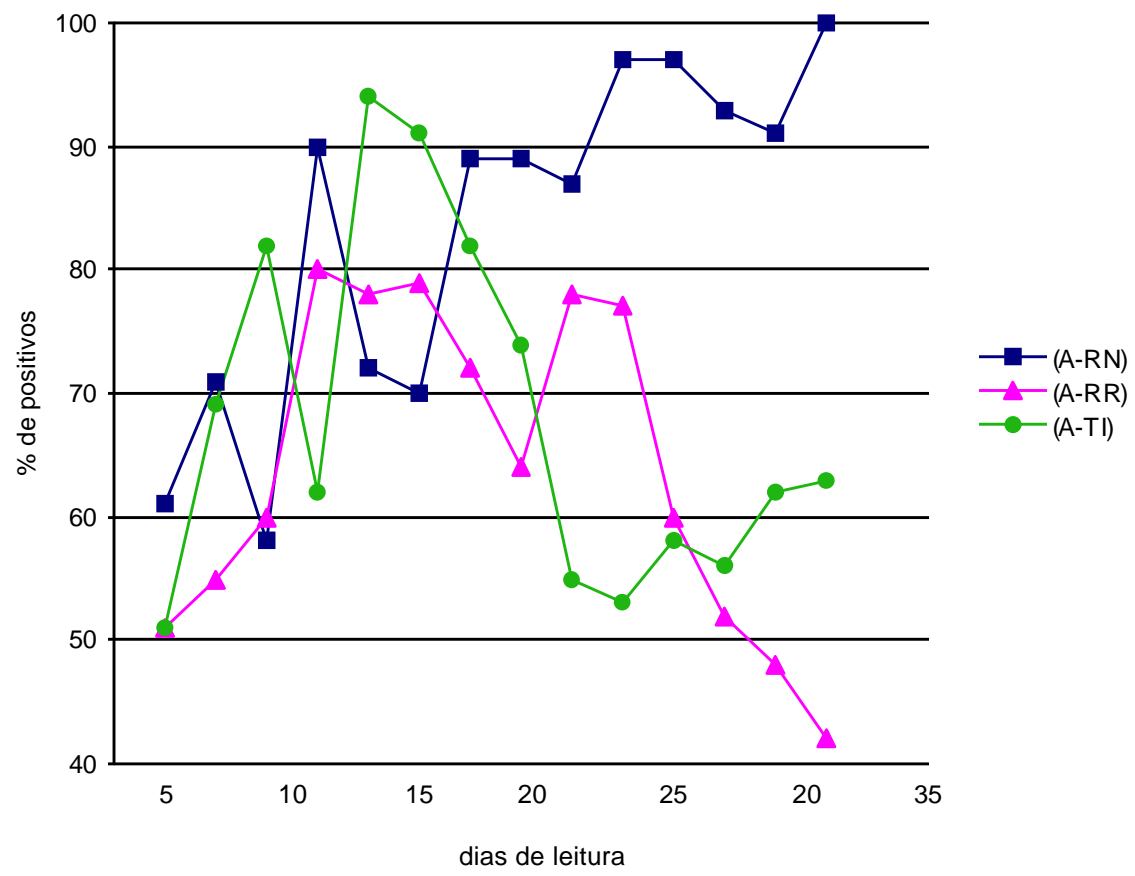

Figura 2 - Curvas médias relativas aos xenodiagnósticos realizados com a cepa AMJM de Trypanosoma cruzi e os triatomíneos Rhodnius neglectus $(R N)$, Rhodnius robustus $(R R)$ e Triatoma infestans $(\mathrm{Tl})$. 


\section{DISCUSSÃO}

Para que o resultado do xenodiagnóstico seja considerado positivo, o encontro de apenas uma única forma de tripomastigota é necessário. Assim, ao realizar as respectivas leituras nas ninfas de triatomíneos utilizadas no experimento, encontramos $71,9 \%$ de ninfas infectadas com a cepa AMJM de T. cruzi, enquanto para a cepa Y, obtivemos $85,2 \%$, podendo-se concluir que a possibilidade de erro quanto ao resultado é menor quando se trata de uma cepa que apresenta alta parasitemia como é o caso da cepa Y.

Analisando todos os resultados obtidos com os xenodiagnósticos realizados neste trabalho, observa-se comportamentos diferenciados das duas cepas de T. cruzi estudadas para as três espécies de triatomíneos. Assim, com a cepa Y, nota-se um padrão mais homogêneo do número de insetos positivos durante os dias examinados e percebe-se, apesar de discreto, uma melhor susceptibilidade dessa cepa com o $R$. robustus (Figura 1), onde os níveis de positividade variaram pouco após o primeiro pico máximo, mantendose praticamente em um platô, contrariando algumas pesquisas realizadas com essas espécies de triatomíneos, onde os autores ${ }^{21} 22$, trabalharam com xenodiagnóstico natural e essas espécies de triatomíneos infectando-os com a cepa Y, e encontraram o T. infestans mais susceptível, seguido pelo $R$. neglectus e $R$. robustus.

Mello e Chiarini ${ }^{10}$, estudando a susceptibilidade de $T$. sordida e $R$. neglectus à infecção pela cepa $Y$ de Trypanosoma cruzi, encontraram as ninfas de $3^{\circ}$ estádio de $R$. neglectus pouco susceptíveis a essa cepa, com níveis de positividade de até $25 \%$.

Silva et $\mathrm{al}^{20}$, trabalhando com xenodiagnóstico artificial e pacientes chagásicos na fase aguda da doença de Chagas, notaram que o $R$. neglectus era o mais susceptível seguido pelo $R$. robustus e T. infestans.

Em 1982, Perlowagora-Szumlewicz e Muller ${ }^{13}$, trabalhando com cobaios infectados na fase aguda da doença com a cepa $Y$, compararam a susceptibilidade de nove espécies de triatomíneos, entre eles o $R$. neglectus e T. infestans e encontraram índices de infecção parasitária superiores no $R$. neglectus quando comparado ao T. infestans, diferente do encontrado neste trabalho onde o $R$. neglectus e T. infestans desenvolveram um comportamento semelhante quando infectados pela cepa $\mathrm{Y}$.

Com relação à cepa $A M J M$, observou-se variações do número de insetos positivos para cada espécie de triatomíneo estudada (Figura 2), encontrando melhor susceptibilidade com o $R$. neglectus, seguido pelo T. infestans e $R$. robustus. A cepa AMJM foi utilizada neste experimento por apresentar características diversas da cepa Y de T. cruzi, pois determina parasitemia e letalidade baixas em camundongos Swiss, embora não tenha sido estudada por outros autores.

O melhor período para a leitura do xenodiagnóstico artificial foi observado entre 0 $11^{\circ}$ e $19^{\circ}$ dias para a cepa $Y$ e entre o $11^{\circ}$ e $15^{\circ}$ dias para a cepa AMJM, quando obteve-se o maior número de insetos parasitados nas três espécies de triatomíneos estudadas. Pesquisas anteriores ${ }^{15}$ que buscavam diminuir o tempo demandado para execução da leitura dos xenodiagnósticos, estabeleceram períodos entre o $15^{\circ}$ e $25^{\circ}$ dias pós infecção em casos de doença de Chagas aguda. Forattini et $\mathrm{al}^{8}$, encontraram o período ideal no $21^{\circ}$ dia pós infecção, sendo o material fecal obtido por compressão abdominal.

Ao compararmos a susceptibilidade das três espécies de triatomíneos estudadas com as duas cepas de T. cruzi, observamos que o R. neglectus mostrou boa susceptibilidade quando infectado tanto pela cepa Y quanto AMJM de T. cruzi. O T. infestans demonstrou melhor susceptibilidade quando infectado pela cepa $Y$ do que pela AMJM de T. cruzi e o $R$. robustus demonstrou boa susceptibilidade quando infectado pela cepa Y e baixa pela cepa AMJM de T. cruzi.

\section{REFERÊNCIAS BIBLIOGRÁFICAS}

1. Barretto AC, Marsden PD, Cuba CC, Alvarenga NJ. Estudo preliminar sobre o emprego de Dipetalogaster maximus (UHLER,1894) (Triatominae) na técnica do xenodiagnóstico em forma crônica de doença de Chagas. Revista Instituto de Medicina Tropical de São Paulo 20:183-189, 1978.

2. Brener Z, Gazzinelli RT. Immunological control of Trypanosoma cruzi infection and pathogenesis of Chagas' disease. International Archives of Allergy and Immunology 114:103-110, 1997.

3. Brumpt E. O xenodiagnóstico: aplicação ao diagnóstico de algumas infecções parasitárias e em particular a trypanosomose de Chagas. Annaes Paulistas de Medicina e Cirurgia 3:97-102, 1914.

4. Campos R, Amato Neto V, Matsubara L, Moreira AAB, Pinto PLS. Estudo sobre o xenodiagnóstico "in vitro". I. 
Escolha de anticoagulante e de membrana. Revista do Hospital de Clínicas da Faculdade de Medicina de São Paulo 43:101-103, 1988.

5. Cerisola JA, Rohwedder R, Segura EL, Del Prado CE, Alvarez M, Martini GJW. El xenodiagnóstico. Secretaria do Estado de la Salud Publica, 1974.

6. Chiari E, Dias JCP, Lana M, Chiari CA. Hemocultures for the parasitological diagnosis of human chronic Chagas' disease. Revista da Sociedade Brasileira de Medicina Tropical 22:19-23, 1989.

7. Costa CH, Costa MT, Weber JN, Gileks GF, Castro C, Marsden PD. Skin reactions to bug bites as a result of xenodiagnosis. Transactions of the Royal Society of Tropical Medicine and Hygiene 75: 405-408, 1981.

8. Forattini OP, Cotrim M, Galati EA, Sarzana SB, Cruz CF, Vanditeren NHS, Gothieb SLD. Estudo sobre a utilização de Rhodnius neglectus para xenodiagnóstico realizados em marsupiais (Didelphis). Revista de Saúde Pública 10:335-343, 1976.

9. Guariento ME. Diagnóstico sorológico da Doença de Chagas em serviço de hemoterapia- aspectos controversos. Revista da Sociedade Brasileira de Medicina Tropical 27(supl II):124-126, 1994.

10. Mello DA, Chiarini C. Suscetibilidade dos diferentes estádios evolutivos de Triatoma sordida (Stal, 1859) e de Rhodnius neglectus (Lent, 1954) à infecção pelo Trypanosoma cruzi. Revista Brasileira de Biologia 40:327334, 1980.

11. Mott KE, França JT, Barrett TV, Hoff R, Oliveira TS, Sherlock IA. Cutaneous allergic reactions to Triatoma infestans after xenodiagnosis. Memórias do Instituto Oswaldo Cruz 75:3-10, 1980.

12. Panameño JAP. Estudo comparativo entre o xenodiagnóstico artificial e o natural na fase crônica da Doença de Chagas. Dissertação de Mestrado. Faculdade de Ciências da Saúde, Universidade de Brasília, Brasília, 1996.

13. Perlowagora-Szumlewicz A, Muller CA. Studies in search of a suitable experimental insect model for xenodiagnosis of hosts with Chagas' disease. 1. Comparative xenodiagnosis with nine triatominae species of animals with acute infections by Trypanosoma cruzi. Memória do Instituto Oswaldo Cruz 77:37-53, 1982.

14. Perlowagora-Szumlewicz A, Muller CA, Moreira CJ. Studies in a search of a suitable experimental insect model for xenodiagnosis of hosts with Chagas' disease. 3. On the interaction of vector species and parasite strain in the reaction of bugs to infection by Trypanosoma cruzi. Revista de Saúde Pública 22:390-400, 1988.

15. Rodwedder RW, Prado CE, Cerisola JÁ, Rebosolan JB. Aportes al método del examen del xenodiagnóstico previo licuado de los triatominos. Boletim Chileno de Parasitologia 25:106-110, 1970.

16. Romaña C, Gil J. Xenodiagnóstico artificial. Annaes del Instituto de Medicina Regional (Tucuman) 2:57-60, 1947.

17. Rosa JA, Belda Neto FM, Buainain A, Giazzi JF, Martini AS, Cunha Filho C, Ribeiro CA. Características de cepas de Trypanosoma cruzi isoladas de chagásicos crônicos. In: XXXIV Jornada Farmacêutica Prof ${ }^{a}$ Deise Pasetto Falcão. Faculdade de Ciências Farmacêuticas de Araraquara-SP, p. 18, 1987.

18. Santos AH, Silva IG, Rassi A. Estudo comparativo entre o xenodiagnóstico natural e o artificial em chagásicos crônicos. Revista da Sociedade Brasileira de Medicina Tropical 28:367-373, 1995.

19. Silva IG. Dispositivo para a realização do xenodiagnóstico artificial. Revista Patologia Tropical 20:35-38, 1991.

20. Silva IG, Luquetti AO, Silva HHG. Importância do método de obtenção das dejeções dos triatomíneos na avaliação da susceptibilidade triatomínica para Trypanosoma cruzi. Revista da Sociedade Brasileira de Medicina Tropical 26:19-24, 1993.

21. Silva IG, Nakano H, Silva HHG, Nakano R. Estudo da susceptibilidade de diferentes espécies de triatomíneos (Hemiptera, Reduviidae) ao Trypanosoma cruzi (Kinetoplastida, Trypanosomatidae). Annaes da Sociedade Entomologia Brasil 23:495-501, 1994.

22. Silva IG, Silva HHG. Susceptibilidade de 11 espécies de triatomíneos (Hemiptera, Reduviidae) à cepa "Y" de Trypanosoma cruzi (Kinetoplastida, Trypanosomatidae). Revista Brasileira de Entomologia 37:459-463, 1993.

23. Silva LHP, Nussenzweig V. Sobre uma cepa de Trypanosoma cruzi altamente virulenta para o camundongo branco. Folia Clínica e Biológica 20:191208, 1953.

24. Souza HBWT, Moreira AAB, Matsubara L, Campos R, Amato Neto V, Pinto PLS, Takiguti CK. Estudo sobre o xenodiagnóstico "in vitro". II. Comparação com o xenodiagnóstico "in vivo". Revista do Hospital de Clínicas da Faculdade de Medicina de São Paulo 43:165-167, 1988.

25. Teo SK, Cheah JS. Severe reaction to the bite of the triatomid bug (Triatoma rubrofasciata) in Singapore. Journal of Tropical Medicine and Hygiene 76:161-162, 1973.

26. Torno CO, Soares V, Vexenat A, Cuba CC, Barreto AC, Alvarenga NJ, Marsden PD. A case study of xenodiagnosis. Revista do Instituto de Medicina Tropical de São Paulo 23:229-232, 1981.

27. Urribarri RS. El xenodiagnóstico. Experiência personal em 100 casos de enfermedad de Chagas crônica. Kasmera, Venezuela 3:167-225, 1970. 